\title{
La construction de locomotives dans les ateliers des anciennes compagnies
}

The construction of locomotives in the workshops of the former companies

\section{Gabriel Curtet}

\section{OpenEdition}

\section{Journals}

Édition électronique

URL : https://journals.openedition.org/rhcf/1784

DOI : $10.4000 /$ rhcf. 1784

\section{Éditeur}

Rails \& histoire

\section{Édition imprimée}

Date de publication : 1 décembre 2003

Pagination : 326-352

ISBN : 0996-9403

ISSN : 0996-9403

\section{Référence électronique}

Gabriel Curtet, "La construction de locomotives dans les ateliers des anciennes compagnies », Revue d'histoire des chemins de fer [En ligne], 28-29 | 2003, mis en ligne le 30 décembre 2014, consulté le 22 avril 2022. URL : http://journals.openedition.org/rhcf/1784 ; DOI : https://doi.org/10.4000/rhcf.1784 


\section{Gabriel CURTET}

\section{La construction de locomotives dans les ateliers des anciennes compagnies}

Cette étude concerne les ateliers des anciennes compagnies françaises qui intégraient des dépôts. Elle est le résultat de recherches menées par trois passionnés d'histoire ferroviaire : le docteur A. Mühl (NeustattPalatinat) ; J. Gillot, ex-cadre SNCF, élève d'André Chapelon, ingénieur des Arts et Manufactures ; moi-même, Gabriel Curtet, ancien professeur de l'enseignement technique.

Ce que je souhaite vous faire partager à travers cette présentation des constructions de locomotives réalisées par les compagnies ellesmêmes, c'est notre amour des machines.

À l'image de la Grande-Bretagne, les grandes compagnies françaises furent amenées, pendant presqu'un siècle, à construire intégralement ou par transformation des locomotives à vapeur répondant aux nécessités du service de l’Exploitation.

Pourquoi les compagnies de chemins de fer se sont-elles lancées elles-mêmes dans la construction? Jusqu'en 1914 l'industrie française spécialisée ne pouvait répondre intégralement aux commandes des grandes compagnies. On peut citer, parmi les constructeurs français : Anzin, Benet, Halette, Moteau, Pauwels, Stehelin et Huber, que l'on retrouvera plus loin parmi les fournisseurs de la Compagnie de l'Est, Gouin, Cavé, Buddicom, Parent et Schaken, Cail, Schneider. L'importation en revanche était jugée trop coûteuse, du moins au XIx ${ }^{e}$ siècle ; par ailleurs, les machines étrangères pouvaient rester à l'atelier faute de pièces de rechange et les contrats d'entretien étaient souvent difficiles à faire appliquer. La situation changea néanmoins, notons-le, au début du $\mathrm{xx}^{\mathrm{e}}$ siècle : à partir de 1906, les compagnies furent amenées à passer de nombreuses commandes aux constructeurs étrangers. La Compagnie de l'Est et le PLM se tournèrent vers l'Allemagne, l'Ouest-État vers l'Italie. Entre 1872 et 1914, 600 unités furent ainsi commandées en Allemagne. Les États-Unis furent ensuite, à la suite de missions d'études effectuées par de nombreux ingénieurs français puis du fait de la Première Guerre mondiale, les destinataires de commandes d'abord timides puis très importantes entre 1914 et 1922, car les besoins correspondaient à l'accroissement du trafic voyageurs et marchandises, en constante progression. Les constructions de machines neuves par les compagnies se sont néanmoins poursuivies et s'étendent de 1846 à 1935 (tabl. 1). 


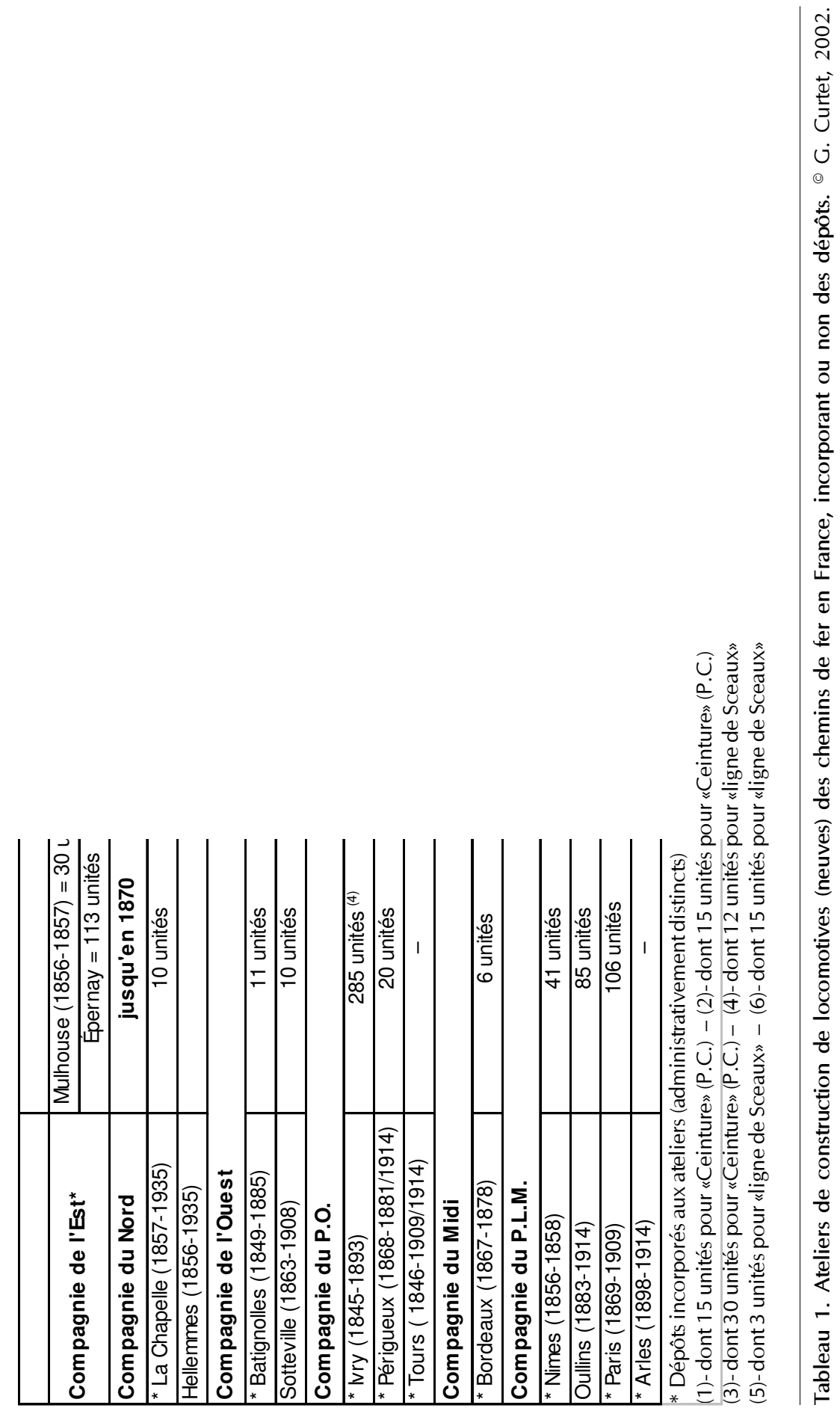


En-dehors de ces motifs conjoncturels extérieurs, cette intégration de la construction par les compagnies exploitantes répondait souvent aux souhaits des conseils d'administration et des ingénieurs qui voulaient éviter tout délai dans le renforcement du parc de machines disponibles. Elle répondait aux impératifs fixés par les conseils d'administration, qui se résumaient le plus souvent à une limitation des coûts. Enfin, la majorité des ingénieurs du service de la Traction était issue des grandes écoles scientifiques françaises - Polytechnique, Arts et Métiers, École Centrale des arts et manufactures. Ce sont ces compétences qu'ils ont mises en œuvre pour concevoir ou transformer les engins de traction mis à leur disposition afin de suivre l'évolution technique mais aussi pour y contribuer dans un climat d'émulation (tabl. 2).

Nous avons évalué l'ensemble des constructions effectuées par les compagnies elles-mêmes à 2834 unités de 1845 à 1935. Ce chiffre est une estimation calculée à partir de nos connaissances, que d'autres pourront corroborer, compléter ou modifier. Par ailleurs, certaines compagnies ayant travaillé pour d'autres - comme le Nord pour la Petite Ceinture (30 unités) et le PO pour le Chemin de fer de Sceaux (15 unités), le nombre total des différentes constructions peut être ramené à 2789 unités.

Chacune des grandes compagnies possédait de grands dépôts dans lesquels étaient réalisés l'entretien courant (révision partielle) et parfois les grandes révisions, dites $R G$, avec « levage intégral ». L'importance de ces dépôts-ateliers alla grandissant face à l'évolution du trafic. C'est ainsi que se créèrent les grands ateliers de construction neuves dont le tableau 1 donne la liste. Nous allons suivre la géographie des réseaux en évoquant, pour chaque compagnie, ces ateliers, les ingénieurs-constructeurs, les locomotives produites enfin.

\section{La Compagnie des chemins de fer de I'Est}

Les deux principaux ateliers sont Mulhouse et Épernay (tabl. 1). Mulhouse a connu un développement précoce des chemins de fer, avec Mulhouse-Thann, ligne plus tard incorporée au réseau de l'Est. La construction de machines à vapeur est caractérisée par la même précocité, stimulée par les besoins des industries textiles, favorisée par l'expérience acquise dans ce domaine (machines de pesage, cardage, tissage), et par la disponibilité d'une main-d'œuvre hautement qualifiée. On peut dire que c'est la finesse de machines textiles qui a permis aux industriels de s'intéresser à la détente dans les distributeurs de cylindres. Les industries privées de construction ferroviaire étaient donc groupées 
Tableau 2. Les ingénieurs-constructeurs de locomotives à vapeur des diverses compagnies françaises, 1846-1938. ํㅜ G. Curtet, 2002.

\begin{tabular}{|c|c|}
\hline \multicolumn{2}{|l|}{ Compagnie des chemins de fer de l'Est } \\
\hline \multirow[t]{10}{*}{ Ateliers d'Épernay } & M. Ed' \\
\hline & O. Sa \\
\hline & M. Vu \\
\hline & L. Res \\
\hline & L. Sal \\
\hline & M. La \\
\hline & F. Dur \\
\hline & A. Lo \\
\hline & M. Pol \\
\hline & M. Wi: \\
\hline \multirow[t]{2}{*}{ Ateliers de Bitschw iller (à l'origine du Mulhouse-Thann) } & J.-J. A \\
\hline & M. St \\
\hline \multicolumn{2}{|l|}{ Compagnie des chemins de fer du Nord } \\
\hline \multirow[t]{10}{*}{ Ateliers de La Chapelle, Hellemmes et Saint-Martin } & M. Le \\
\hline & M. Fol \\
\hline & J. Peti \\
\hline & E. Del \\
\hline & F. Ma \\
\hline & G. $d u$ \\
\hline & G. As \\
\hline & L. $\mathrm{Br} \epsilon$ \\
\hline & G. Co \\
\hline & J. Lar \\
\hline Compagnie des chemins de fer de l'Ouest & \\
\hline
\end{tabular}


(Tableau 2 - suite)

\begin{tabular}{|l|l}
\hline \multicolumn{2}{|c|}{ Com pagnie des chemins de fer de Paris-Orléans } \\
\hline Ateliers d'lvry-le Chevaleret & C. Polon \\
\hline & V. Forqu \\
\hline & E. Polonc \\
\hline & E. Solacı \\
\hline & F.-P. Duk \\
\hline & M. Lacoi \\
\hline & L. de Bo \\
\hline & E. Epina \\
\hline
\end{tabular}

Compagnie des chemins de fer du Midi

\begin{tabular}{|c|c|}
\hline \multirow[t]{6}{*}{ Ateliers de Bordeaux } & M. Sage \\
\hline & M. Laure \\
\hline & M. Millet \\
\hline & M. Moffr \\
\hline & A. Herdr \\
\hline & A. Bachı \\
\hline \multicolumn{2}{|c|}{ Compagnie des chemins de fer du P.O. - Midi (fusion $\epsilon$} \\
\hline \multirow[t]{2}{*}{ Ateliers de Tours } & A. Bachı \\
\hline & M. Cardc \\
\hline \multicolumn{2}{|c|}{ Compagnie des chemins de fer du P.LM. (Paris-Lyon-M } \\
\hline Section Nord (Paris) & M. Lecor \\
\hline \multirow[t]{4}{*}{ Section Sud (Lyon) } & M. de Bc \\
\hline & M. Lecor \\
\hline & G. Marié \\
\hline & A. Henry \\
\hline
\end{tabular}




\begin{tabular}{|c|c|c|}
\hline 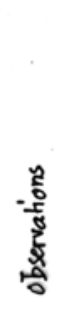 & \multicolumn{2}{|c|}{ 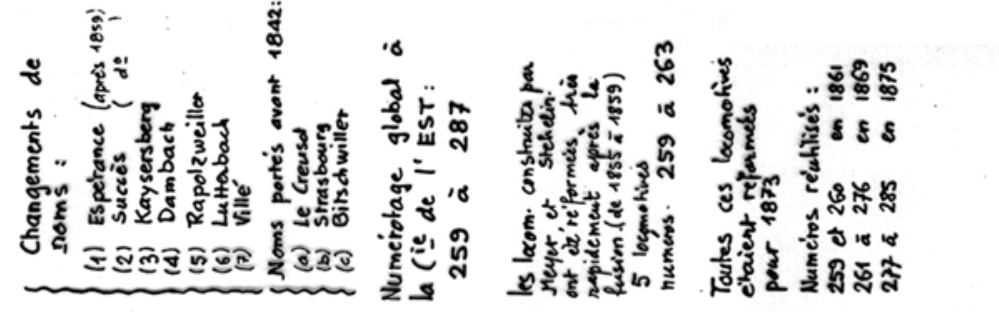 } \\
\hline$\frac{\delta}{8} 8 \frac{8}{\frac{5}{6}}$ & 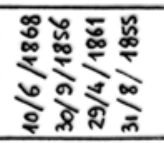 & 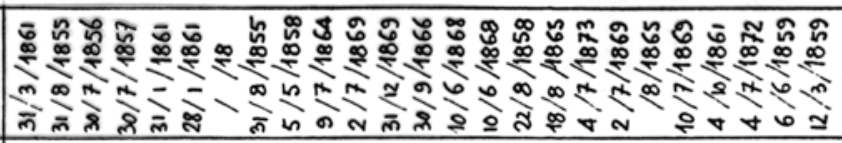 \\
\hline 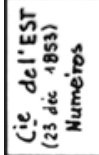 & 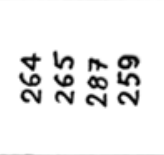 & 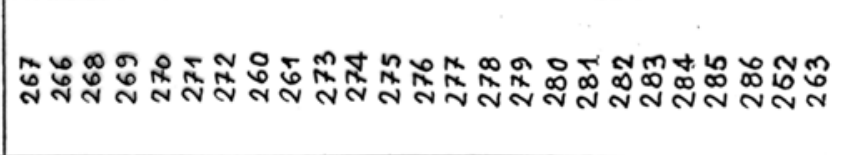 \\
\hline 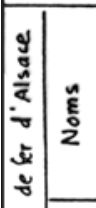 & 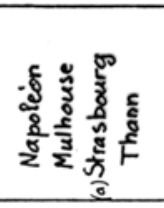 & 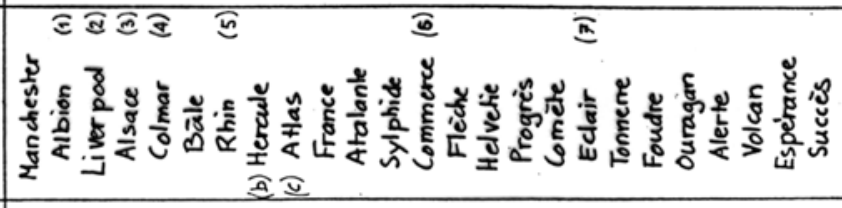 \\
\hline 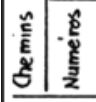 & н日月』1 & 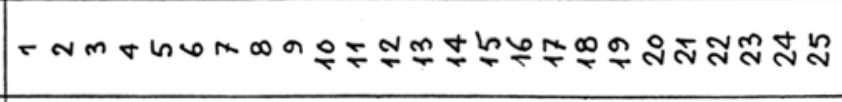 \\
\hline م. & 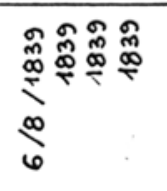 & 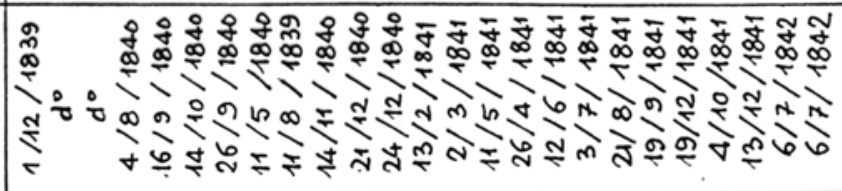 \\
\hline 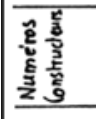 & $-\alpha \underline{\Gamma} \sim$ & ํํำ \\
\hline 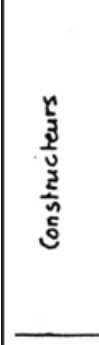 & 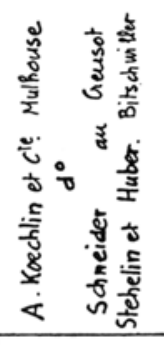 & 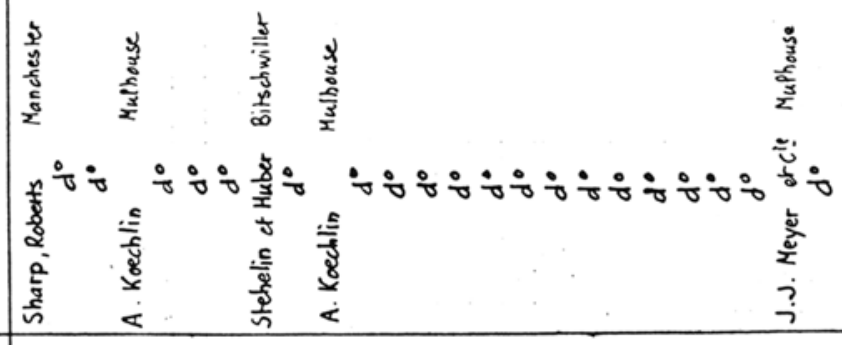 \\
\hline 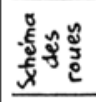 & $\underset{F}{F} \underset{T}{\gtrless}$ & 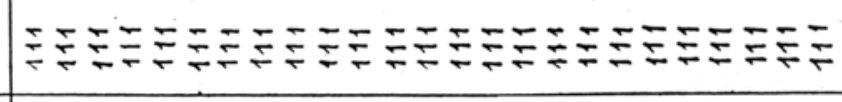 \\
\hline 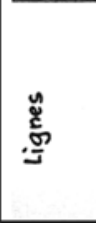 & 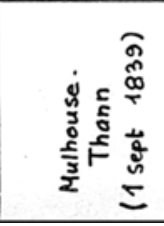 & 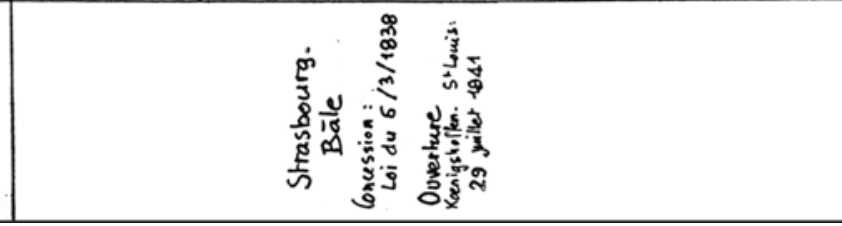 \\
\hline
\end{tabular}

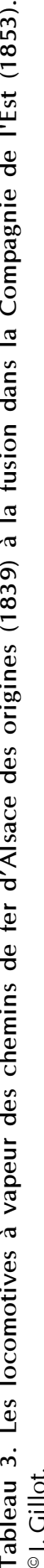




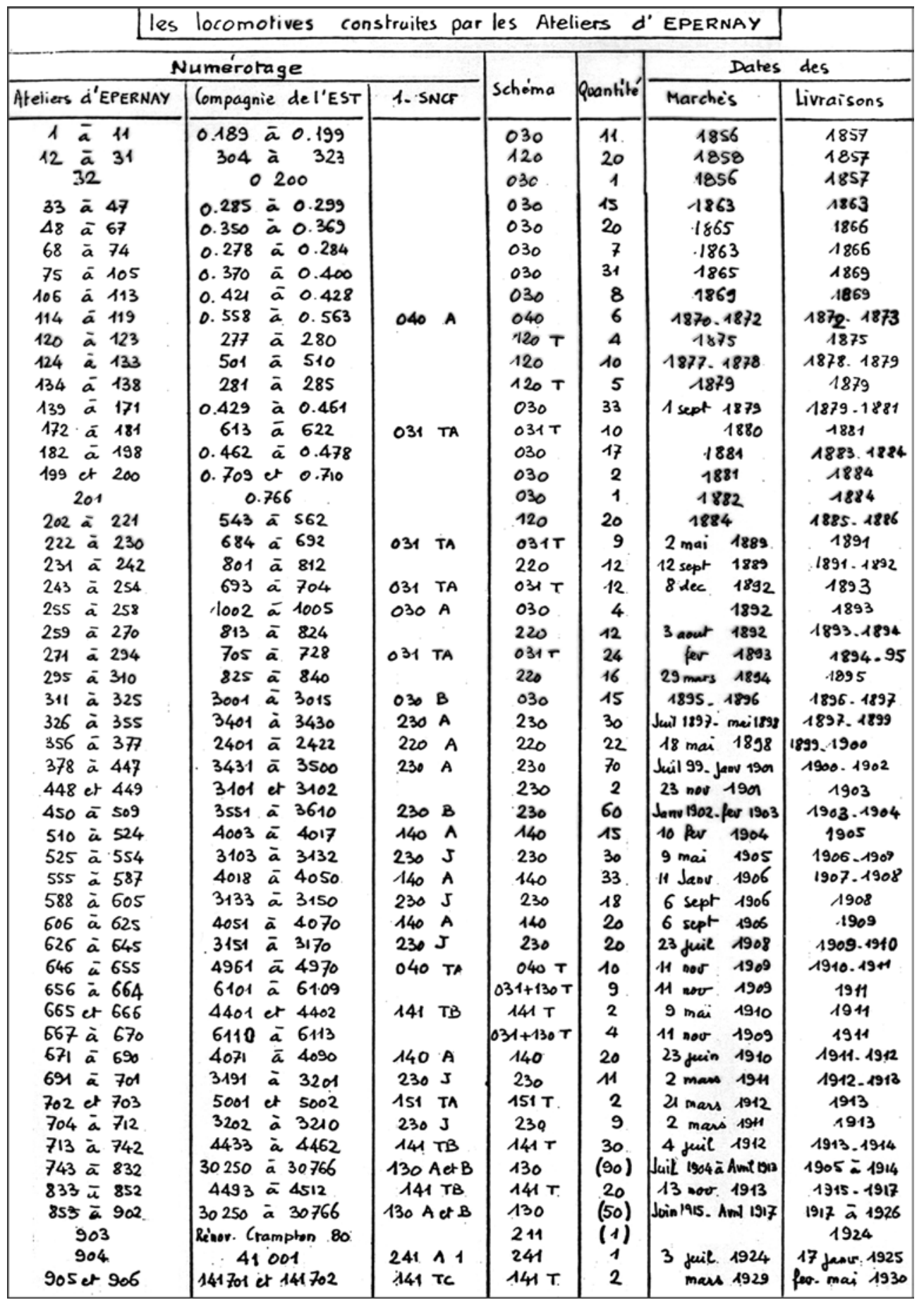

Tableau 4. Les locomotives construites par les ateliers d'Epernay, 1856-1930. ${ }^{\odot} \mathrm{J}$. Gillot. 
autour de Mulhouse, comme Stehelin ou Koechlin. Dans l'inventaire dressé par J. Gillot des machines des chemins de fer d'Alsace avant la fusion qui a formé le réseau de la Compagnie de l'Est (1839-1853) et de leurs constructeurs, on relève les noms de Kœchlin, Schneider, Robert Sharp (machines importées) (tabl. 3). Kœchlin, Stehelin et Huber habitent Bischwiller et Mulhouse, centre de la construction ferroviaire en Alsace. Jean-Jacques Meyer, industriel mulhousien, a créé des machines exceptionnelles. Elles se sont peu développées parce qu'il répondait toujours à des cahiers des charges très particuliers. Pour le PLM il a par exemple construit «la Courbe ». Les innovations techniques qu'il a développées sont étonnantes, à tel point que la Deutsche Bahn a fabriqué en 1952 des machines 050 tender dont la suspension et l'articulation des essieux étaient basées sur un principe de Beugnot.

Cependant, l'essentiel des constructions de la compagnie était concentré à Épernay. L'Atelier central d'Épernay construisit, entre 1856 et 1930, 765 unités, dont pour mémoire les 220 à chaudière Flaman nº01/812 à 879; environ 230 Compound en 1914 (quatre cylindres, surchauffe à partir de 1913); en 1913, les 151 T 5001/5002 à simple expansion et vapeur surchauffée ; en 1925, la première " Mountain » $241 \mathrm{n}^{\circ} 41000$, Compound, quatre cylindres, surchauffe, et les prototypes de 141 tender 141 701/702 à trois cylindres et surchauffe pour la banlieue (tabl. 3,4 ).

En ce qui me concerne, $j$ 'ai toujours été passionné par les hommes qui ont dessiné ces machines. À l'Est nous trouvons (tabl. 2) : Edgar Edwards, premier ingénieur du Matériel qui prit en main la construction de locomotives dans ses ateliers d'Épernay. Ensuite vous avez Oscar Sauvage (1852-1861) (les Sauvage ont été une pépinière d'ingénieurs).

Vuillemin fut un ingénieur qui a étudié le compoundage à deux essieux et qui souvent, du fait que l'Est exploitait le massif vosgien, avait adopté l'innovation du tender moteur (qui vient d'Angleterre et d'Allemagne) (fig. 1). Louis Regray qui vient ensuite s'est aussi beaucoup intéressé aux voitures, à leur chauffage en particulier (son nom a été affecté au type de bouillottes que l'on glissait entre les jambes des voyageurs). C'est à son époque et à celle de Salomon qu'on a entrepris la production des premières Ten Wheels, à leur début machines mixtes pour marchandises et pour voyageurs et non dédiées, comme elles le furent par la suite, aux grandes vitesses. Ensuite, Lancrenon fait aussi partie d'une famille qui a donné plusieurs ingénieurs. Duchâtel a participé au dessin et fait les calculs des «Mountains ». C'est à ses bureaux de dessin que l'on doit une innovation majeure : la plate-forme Mestre, du 


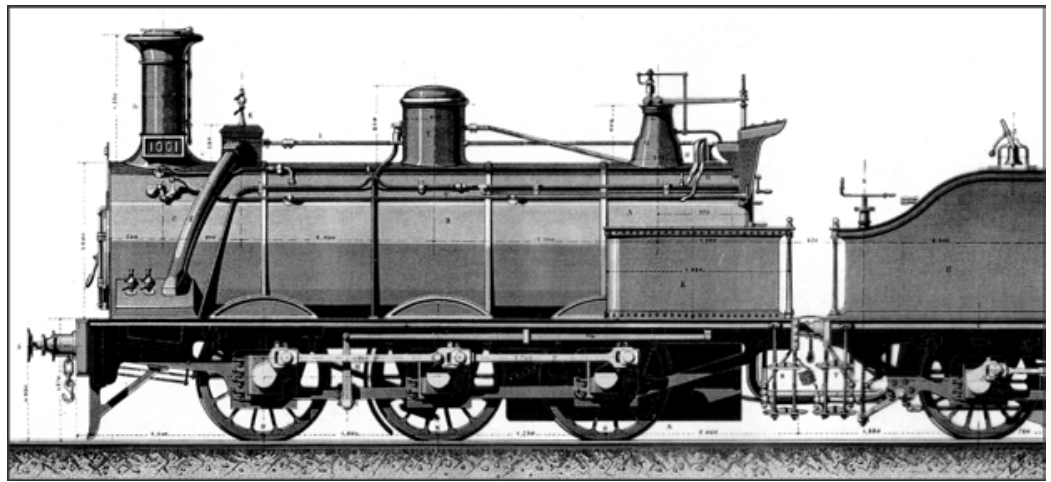

Figure 1. Machine Vuillemin 030 Est, série 1000, 1867, atelier de Graffenstaden (planche extraite de la revue encyclopédique : Le Praticien industriel). Cette locomotive à tender-moteur, à simple expansion et vapeur saturée, est destinée aux trains de marchandises sur des lignes accidentées (rampes de 15\%).

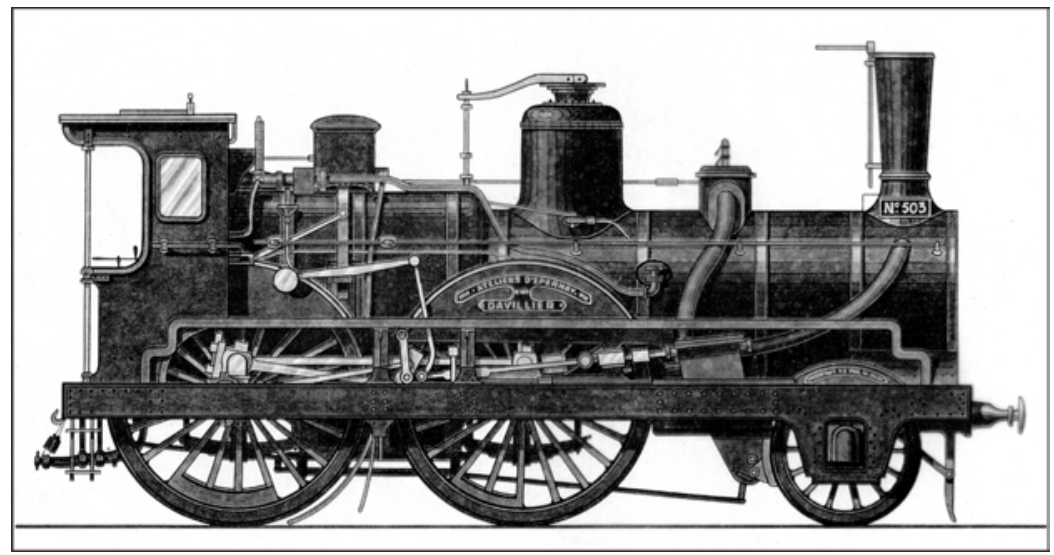

Figure 2. Locomotive $120 \mathrm{n}^{\circ} 503$ de 1878 construite à Épernay, caractérisée par des roues de $2,04 \mathrm{~m}$ de diamètre (que l'on retrouvait, entre autres, sur les carénées allemandes en 1936), destinée aux trains express et rapides de voyageurs. 


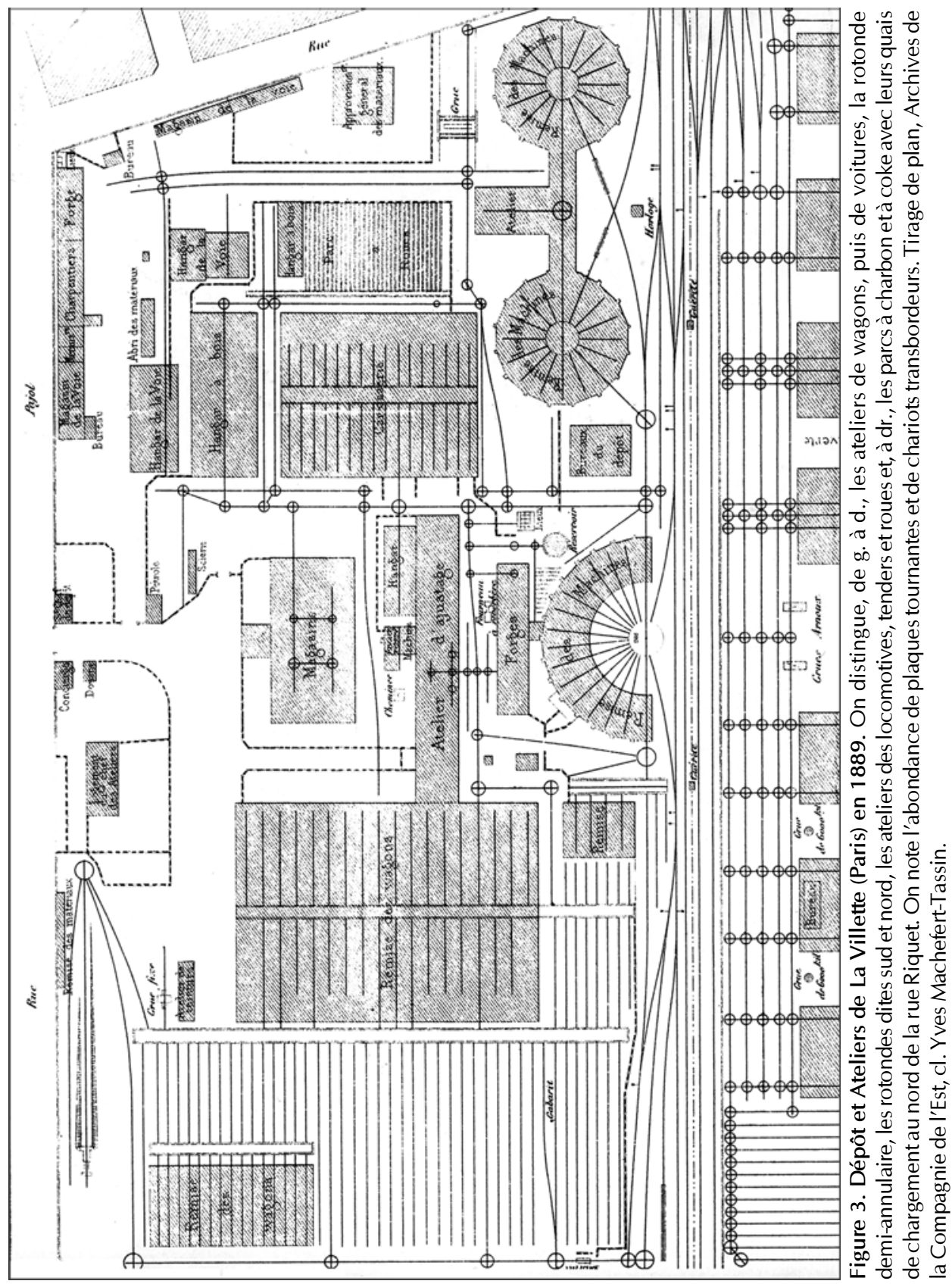


nom de son dessinateur. La plate-forme Mestre a supprimé la césure entre la plate-forme de la machine et la plate-forme du tender, entre lesquelles étaient ballottés mécaniciens et chauffeurs. Elle fut appliquée pour la première fois sur la 41001.

Loizillon a laissé peu de traces, comme Poncet en 1936 dont je ne connais pas la fin de carrière. Wisdorff appartient déjà à la SNCF. C'est sous son autorité que les S16 et les G16 ont été étudiées avec la participation de la SACM. Ce sont, selon moi, les plus les belles réussites esthétiques de la période. Si je n'ai pas abordé l'AL (l'Alsace-Lorraine) c'est à cause des particularités de ce réseau : vous n'ignorez pas toutes les difficultés administratives et techniques intervenues lors de son retour à la France en 1918.

\section{La Compagnie des chemins de fer du Nord}

Le Nord construisit, entre 1857 et 1935, 374 unités à la fois dans ses ateliers de La Chapelle (près de Paris), de Hellemmes (près de Lille) de 1886 à 1935, de Saint-Martin (près de Charleroi), établissement principal du réseau le « Nord-Belge ».

Le site de La Chapelle (fig. 4) a été occupé très tôt, dès 1857, par un atelier de petit entretien et de grandes révisions éventuelles. Tous les grands ingénieurs, quels qu'ils soient, souhaitaient avoir à leur disposition des ateliers et des ouvriers capables de mettre en œuvre les innovations que chacun d'entre eux souhaitait mettre en œuvre ou adapter sur les machines du réseau. La Chapelle n'a cessé cette activité qu'en 1935. Les documents photographiques rares qui suivent montrent l'atelier en exploitation (fig. 5 et 6). On voit d'abord, avec une vue sur le SacréCœur, le grand bâtiment abritant l'atelier avec de nombreux sheds successifs et les hautes cheminées. L'établissement occupait une superficie considérable et s'étendait jusqu'au boulevard des fortifications. La première machine visible est une 222 à chaudière à tubes d'eau : Schneider avait en effet poussé le Nord a expérimenter cette solution. Elle n'a pas fini sa carrière sous cette forme, car de 222 elle est passée à une disposition 230 , très réussie avec une très belle allure et la livrée bien connue du Nord, cette teinte « chocolat» dont on disait que les Rothschild l'avaient importée d'Angleterre, du réseau élégant Brighton and South Coast. On aperçoit à sa gauche une 3500 sans échappement Lemaittre. 


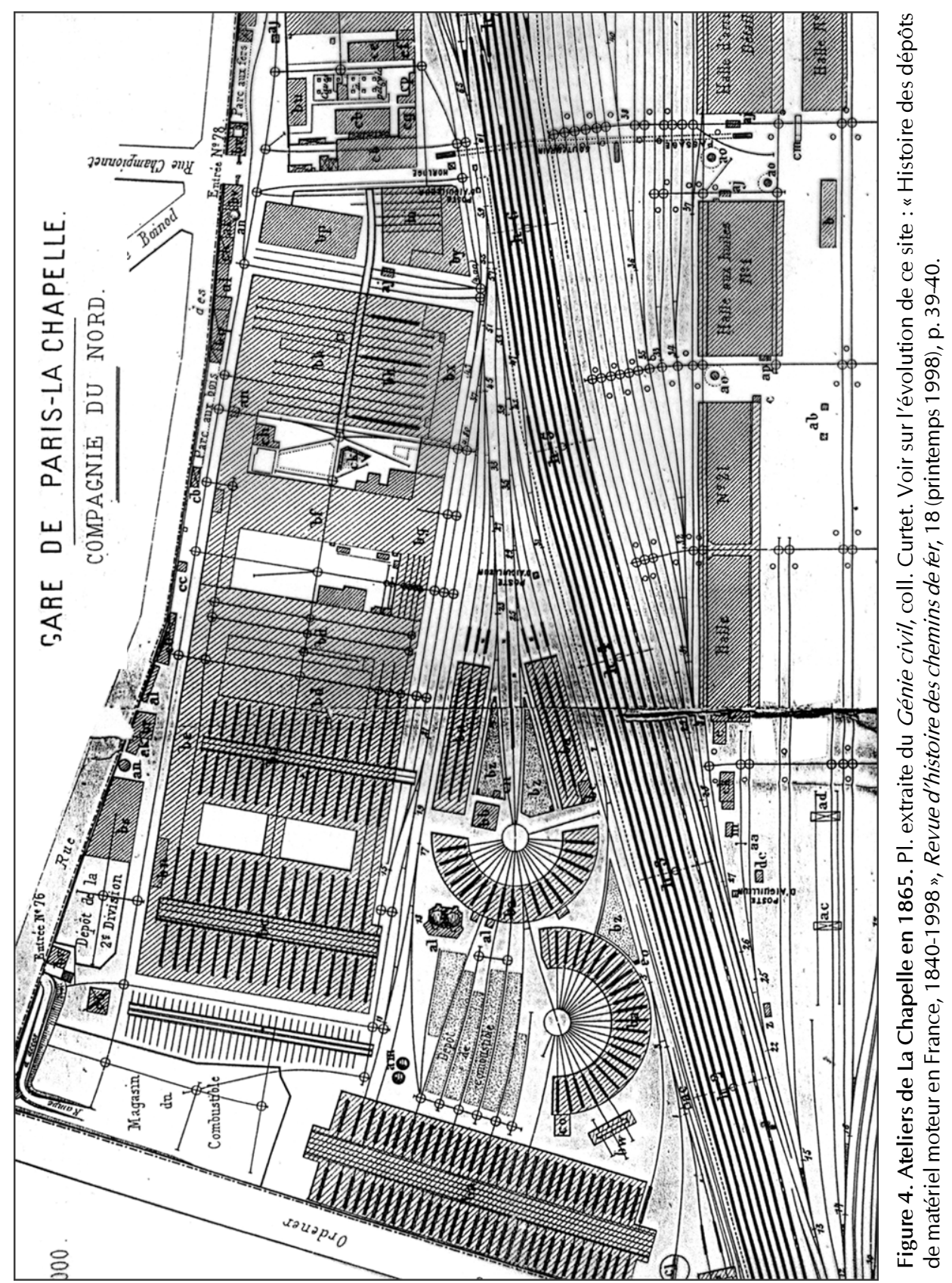




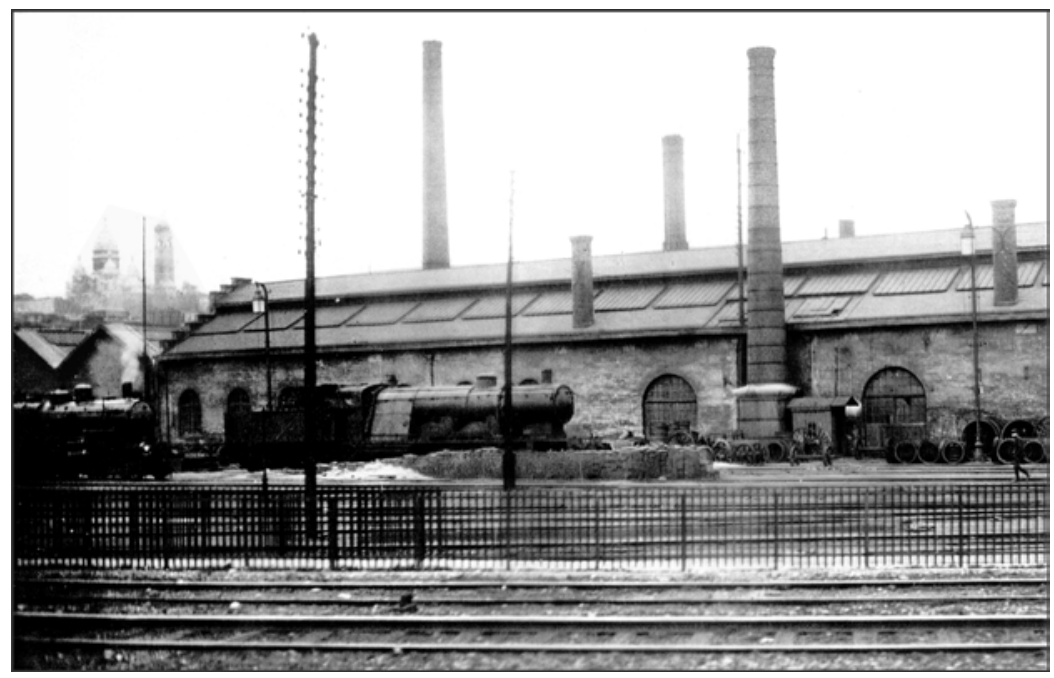

Figure 5. Vue de l'atelier de La Chapelle vers 1910. Cl. Romouil, coll. G. Curtet.

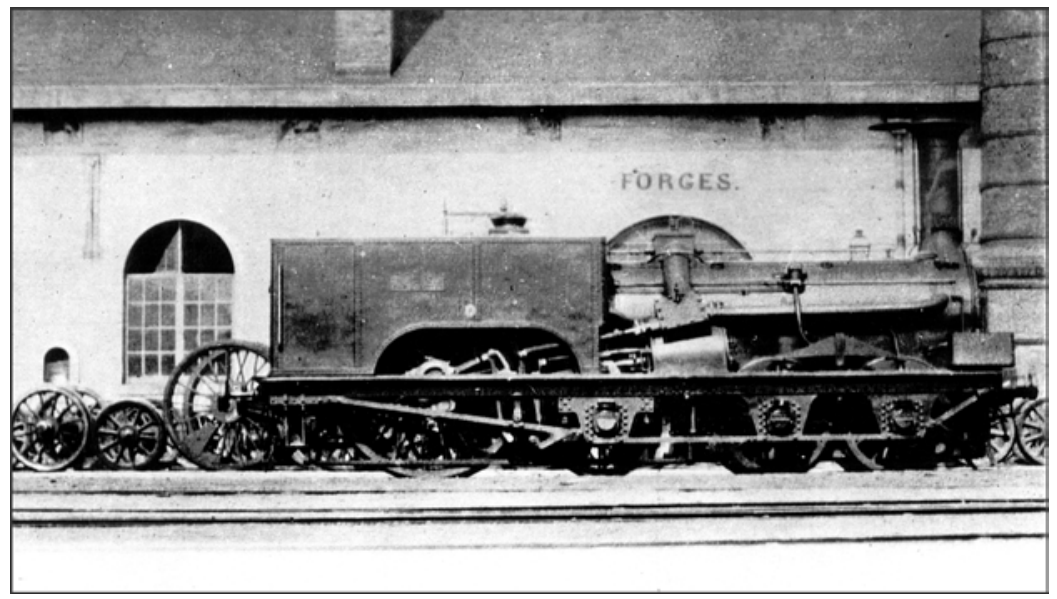

Figure 6. Vue de I'atelier de La Chapelle (Forges) vers 1890. Locomotive Crampton tendérisée à deux cylindres de type $111 \mathrm{~A}$. Cl. Romouil, coll. G. Curtet.

La deuxième illustration (fig. 6) montre la continuité du grand shed et les forges de La Chapelle. Une Crampton tendérisée, la 162 ou la 163 (une série qui a été en service très longtemps), est arrêtée devant l'atelier des roues. 
Hellemmes construisait d'abord du matériel roulant - voitures, wagons - et très peu de machines à vapeur. Puis l'atelier se spécialisa dans les chaudières. Lors des créations de prototypes elle livrait les chaudières à La Chapelle qui continuait la superstructure.

Les ingénieurs du Nord (tabl. 2) sont des célébrités dont le nom est connu en dehors du secteur ferroviaire : Le Châtelier, l'un des piliers scientifiques du développement des chemins de fer, puis Fournel, Jules Petiet, décédé en 1871 à la suite, dit-on, de la défaite, Édouard Delebecque, Ferdinand Mathias de la grande famille des Mathias du Nord. Plus près de nous, Gaston du Bousquet est le vulgarisateur de la Compound à quatre cylindres (en général HP extérieur - BP intérieur) qui a révolutionné le Nord. Georges Asselin a fait dès 1910 ses premiers dessins des Pacifics Nord sous Du Bousquet, les 3.1200 Nord première série. Louis Brevillié était en fonctions pendant la période difficile de la reconstruction après 1918. Quant à Georges Collin, il aussi un dessinateur et un ingénieur des Pacifics deuxième type, caractérisées par le « cercueil » sur la chaudière et deux dômes encadrant la sablière. Enfin, l'un des plus connus, Jean Lancrenon (1931-1937), a été a l'initiative de l'utilisation courante des autorails Nord.

Les machines remarquables sont :

$1^{\text {re }}$ Compound à quatre cylindres, type 220 (2.101), plans $\mathrm{Du}$ Bousquet ;

$1^{\text {re }}$ Compound à trois cylindres, type 130 (3.101), plans Sauvage ;

$1^{\text {re }}$ Compound à quatre cylindres, type 232 (3.1101 et 2) ;

$1^{\text {re }}$ Compound à quatre cylindres en tandem, type $230 \mathrm{~T}$ (petite ceinture $\mathrm{n}^{\circ} 51$ à 62);

$1^{\text {re }}$ Compound à quatre cylindres, type $031+130 \mathrm{~T}$, type Meyer, Du Bousquet, destinées au trafic houiller.

Enfin, la construction se termina par les 150 à quatre cylindres et à vapeur surchauffée, $\mathrm{n}^{\circ} 5.1201$ à 30 , les $141 \mathrm{~T}$ à deux cylindres et à vapeur surchauffée, $\mathrm{n}^{\circ} 4.1201$ et deux pour la banlieue parisienne. 


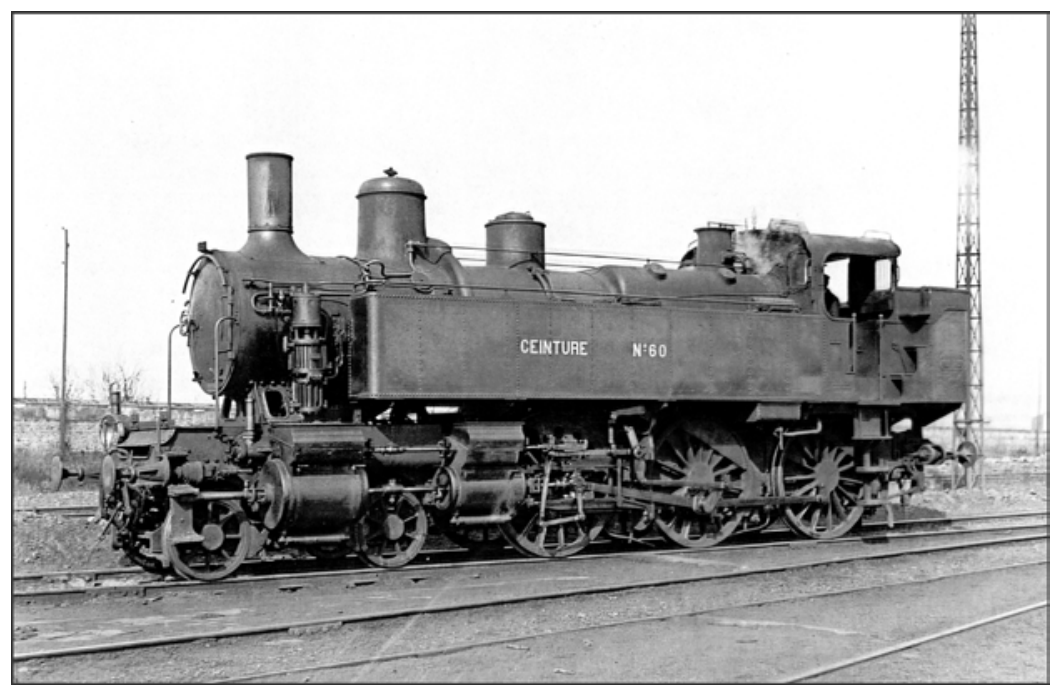

Figure 7. Machine Nord (230 tender) pour la Ceinture parisienne fabriquée à La Chapelle qui présente la particularité de comporter quatre cylindres en "tandem" : les deux cylindres BP-HP sont dans le même axe de chaque côté. C'est en France la seule application de ce procédé sur un matériel courant pour voie normale (système Du Bousquet). Cl. Romouil, 1913, coll. G. Curtet

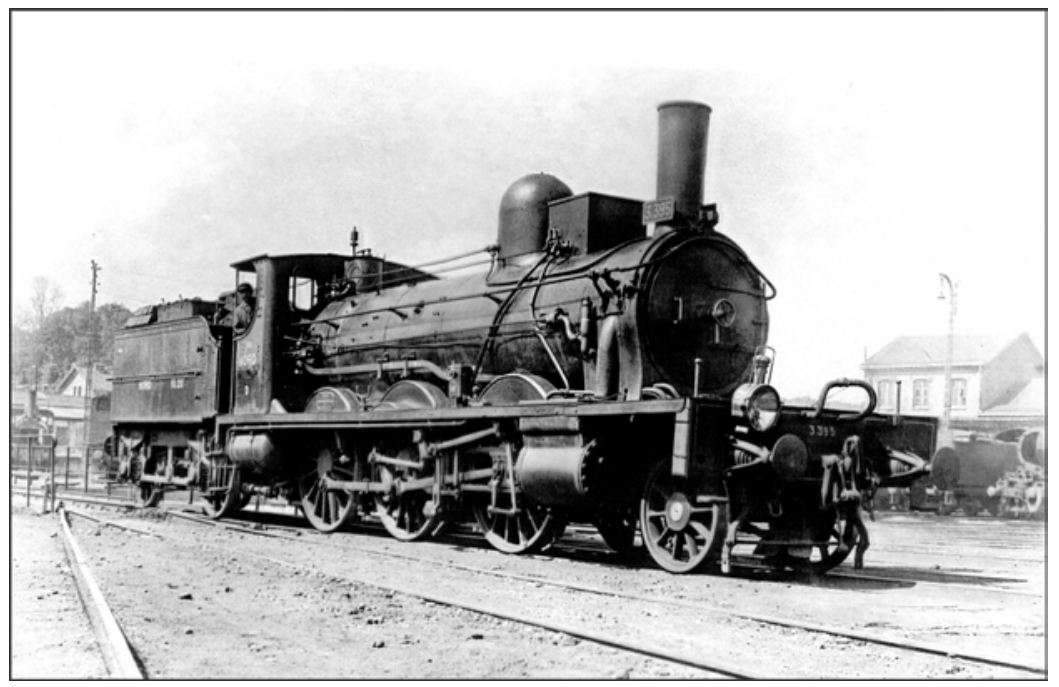

Figure 8. Locomotive pour le transport de marchandises et express de type 130 à trois cylindres compound sur des plans de Sauvage, construite en 1887, ici au dépôt de Creil. Cl. Romouil, vers 1900, coll. G. Curtet. 


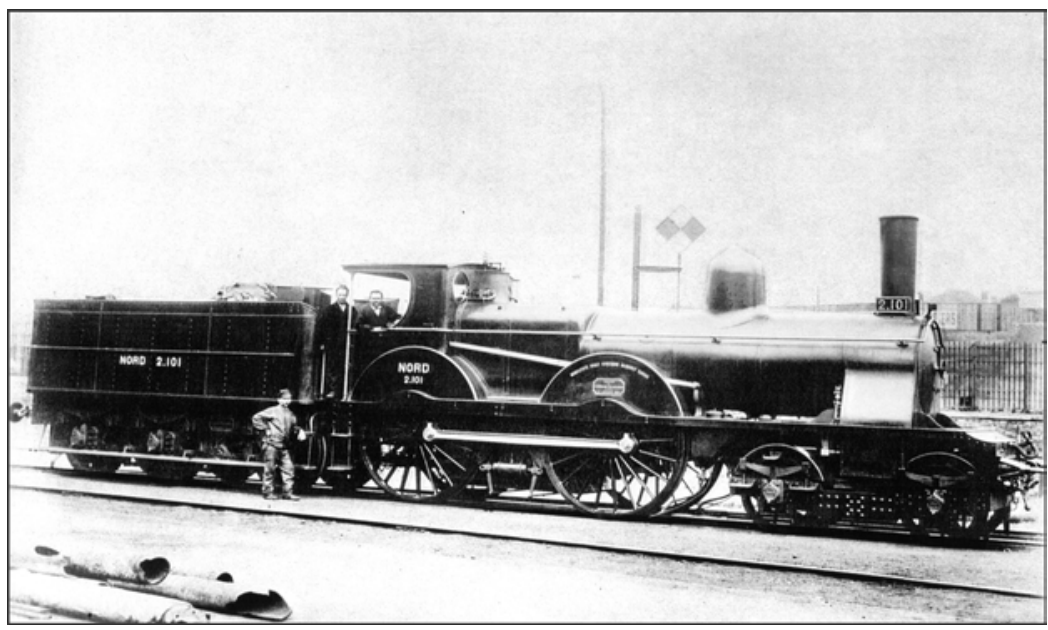

Figure 9. Une des premières machines dessinées par Du Bousquet, I'ancêtre des Du Bousquet 220 à quatre cylindres (2.100), un prototype (ateliers de La Chapelle et d'Hellemmes pour les chaudières). Cliché pris à La Chapelle en 1885. Cl. Compagnie du Nord, coll. G. Curtet.

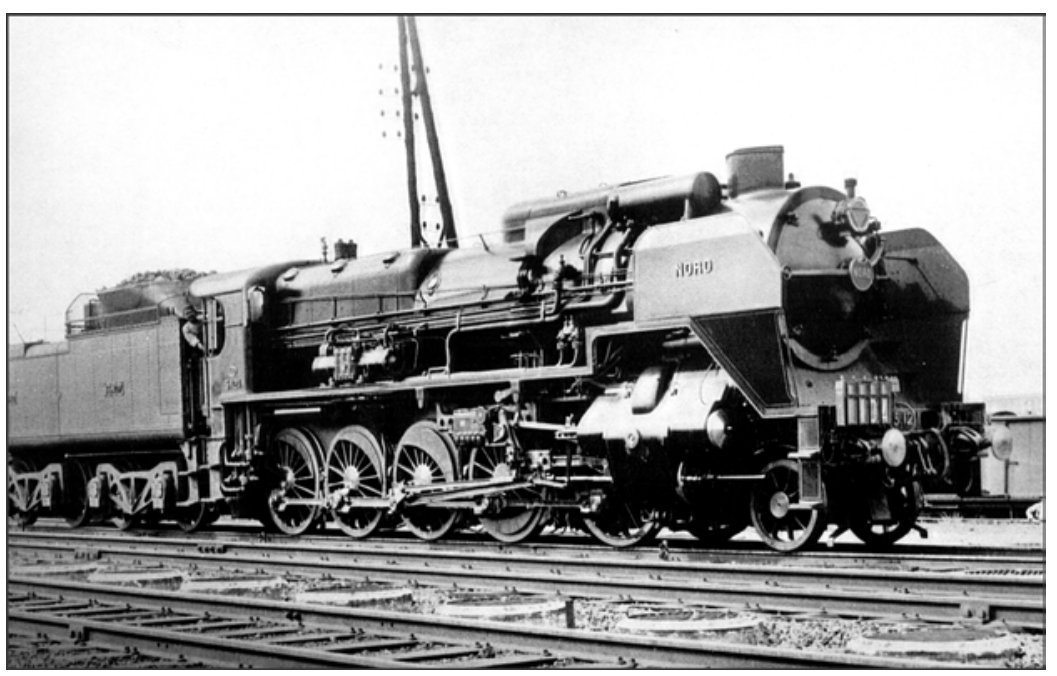

Figure 10. On reconnaît dans la 5.1200 la silhouette des machines dessinées par De Caso (Compound quatre cylindres, vapeur surchauffée, 19321933). Le Nord avait déjà porté le poids des machines à 20 tonnes par essieu, autorisant des poids élevés pour l'adhérence avec des roues de $1,55 \mathrm{~m}$, ce qui constitue une réussite technique exceptionnelle. Un train d'essai tracté par cette machine aurait atteint $150 \mathrm{~km} / \mathrm{h}$, comme certaines 150 de la Deutsch Reichsbahn. Cl. Compagnie du Nord, coll. G. Curtet. 


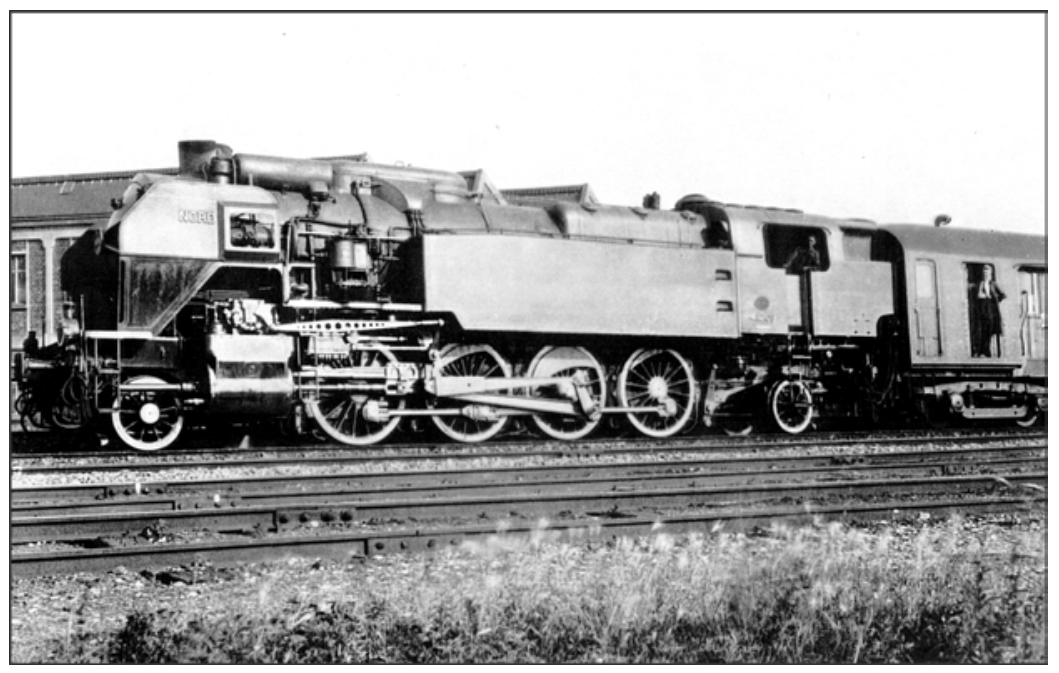

Figure 11. Machine 4.1200 de la Compagnie du Nord, quatre essieux pour 20 tonnes avec une distribution par quatre pistons vannes verticaux, construite pour le service de la banlieue parisienne entre 1932 et 1936 (141 tender, simple expansion, deux cylindres, vapeur surchauffée). Le tender classique (Baume et Marpens pour les Belges) avec sa hotte inclinée est typique du Nord français comme du Nord belge. Cl. Fenino, coll. G. Curtet.

\section{La Compagnie des chemins de fer de l'Ouest}

L'Ouest, avant et après son rachat par l'État en 1908, réalisa entre 1840 et 1920 de nombreuses modifications allant jusqu'à la transformation du matériel de la Compagnie de Saint-Germain à Versailles, origine du réseau, soit aux ateliers des Batignolles (près de Paris) (fig. 12) soit aux ateliers de Sotteville-Quatre-Mares (Rouen).

Dans le premier atelier, le second construisant la chaudière, a été construite la première 231 (Pacific) de l'Ouest en 1908-1909 sur un plan de Dubois, numérotée no 6001/2, Compound à quatre cylindres.

Aux origines de la compagnie c'est aux Batignolles que furent réalisées, sur un plan d'E. Flachat, en 1846, les « Hercules », machines 030 à tender et deux cylindres intérieurs et, trois ans plus tard, en 1849, les «Antée », 031 à tender et deux cylindres extérieurs qui devaient permettre de franchir la rampe de $35 \mathrm{~mm} /$ mètre dite du Pecq à Saint-Germain après une première exploitation par un système à air comprimé. 


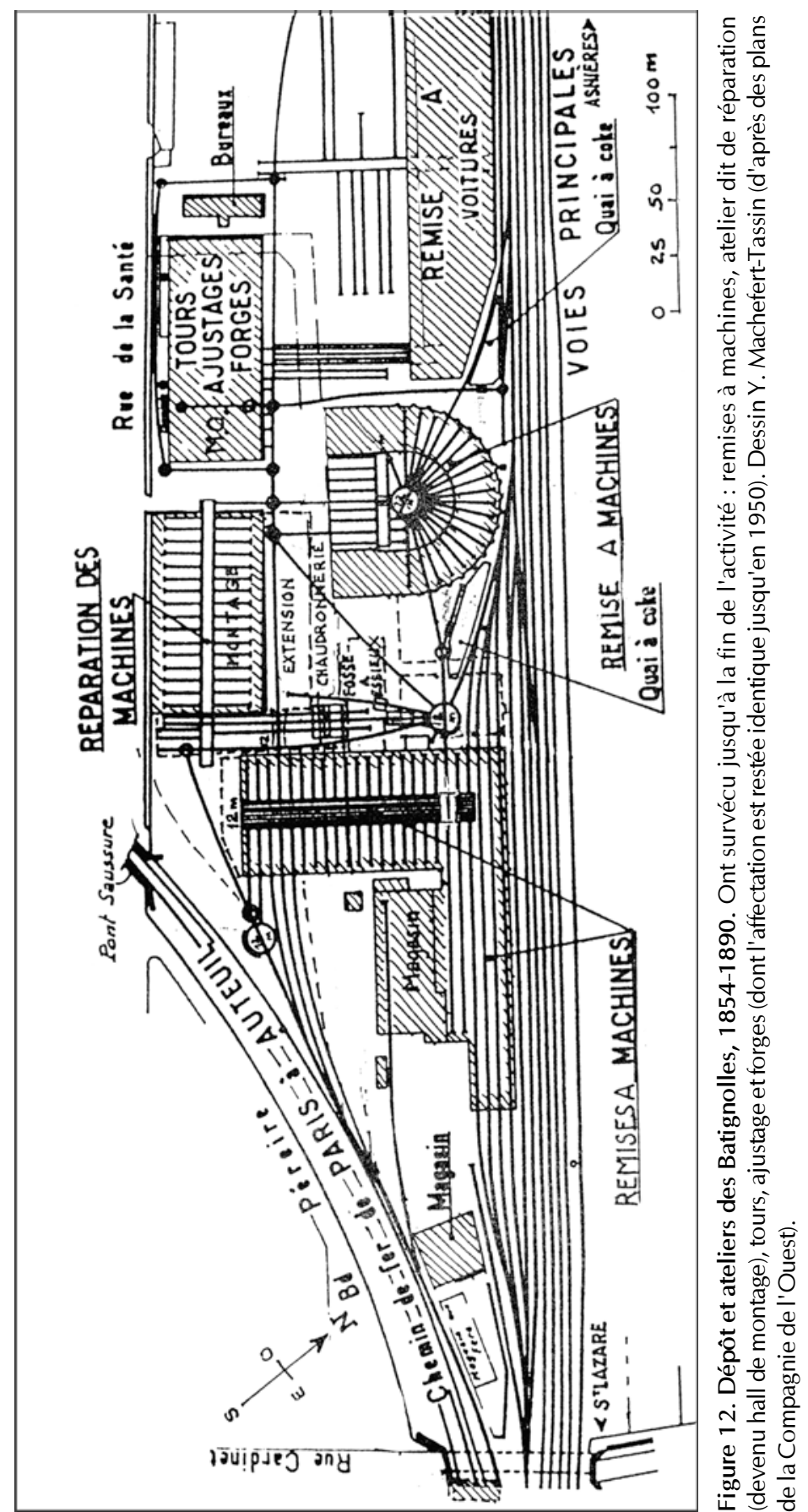




\section{La Compagnie des chemins de fer Paris-Orléans}

Les ateliers parisiens du PO étaient situés à Ivry-Chevaleret, où la période de construction des locomotives s'étend de 1843 à 1900, à Périgueux (1868-1881), à Tours (1909-1940). Sous la remarquable autorité de MM. Polonceau et Forquenot un grand nombre de locomotives furent construites ou re-construites. Ainsi la première $050 \mathrm{~T}$ à deux cylindres d'Europe (1867), dite «Cantal » (1201 à 1203) fut construite aux Atelier d'Ivry et toutes les locomotives de la ligne de Sceaux : 1A1-1A1T et 1B1-1B1T héritées du système d'exploitation Arnoux furent mises à la norme moderne de la banlieue PO. Ensuite, E. Solacroup entreprit, en suivant les idées de De Glehn, de nombreuses constructions nouvelles avec le concours de l'industrie privée, de 1909 à 1914. On retient les 221 à grande surface de grille de la ligne de Bordeaux auxquelles succèdent en 1907 la 231, puis les 230 (4001) dont certaines avec des adaptations en trois types réalisées par les Ateliers du PO à Tours, par exemple les 2303.221 à 3.235 (Tours, 1913). Ces machines étaient beaucoup plus puissantes, de 12 à $43 \%$, que les précédentes.

Entre-temps le parc de traction $\mathrm{PO}$ passait de 523 engins à 1348 avec Forquenot en 1860, à 1415 en 1899 avec Polonceau et à 1589 dès 1907 avec Solacroup. Malgré cela, le réseau paraissait pauvre avec une machine pour 4,4 km, comme le Midi, alors que le PLM atteignait un coefficient de 3,2.

Ce fut sous l'autorité de M. Épinay qu'A. Chapelon put réaliser à Tours la transformation complète de la 231 n 3566 C 45 (prototype) ainsi que la transformation et la livraison aux Chemins de fer de l'Est et du Nord des 231 dites « Chapelon » suivies du prototype de 240 4701/ 712 pour le $\mathrm{PO}$ et, plus tard, les $240 \mathrm{P} \mathrm{SNCF}$; enfin pour terminer, la 160 A livrée en juin 1940.

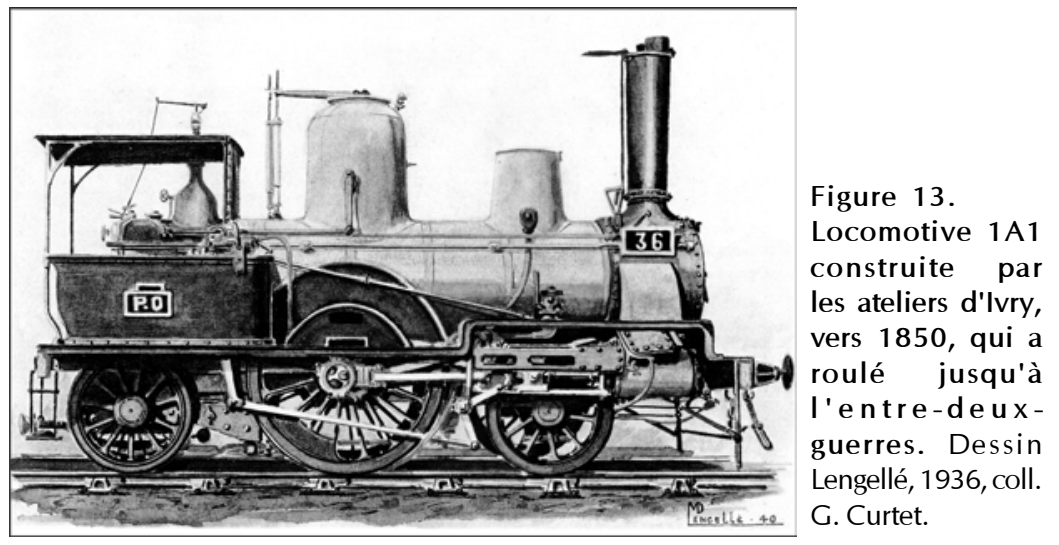




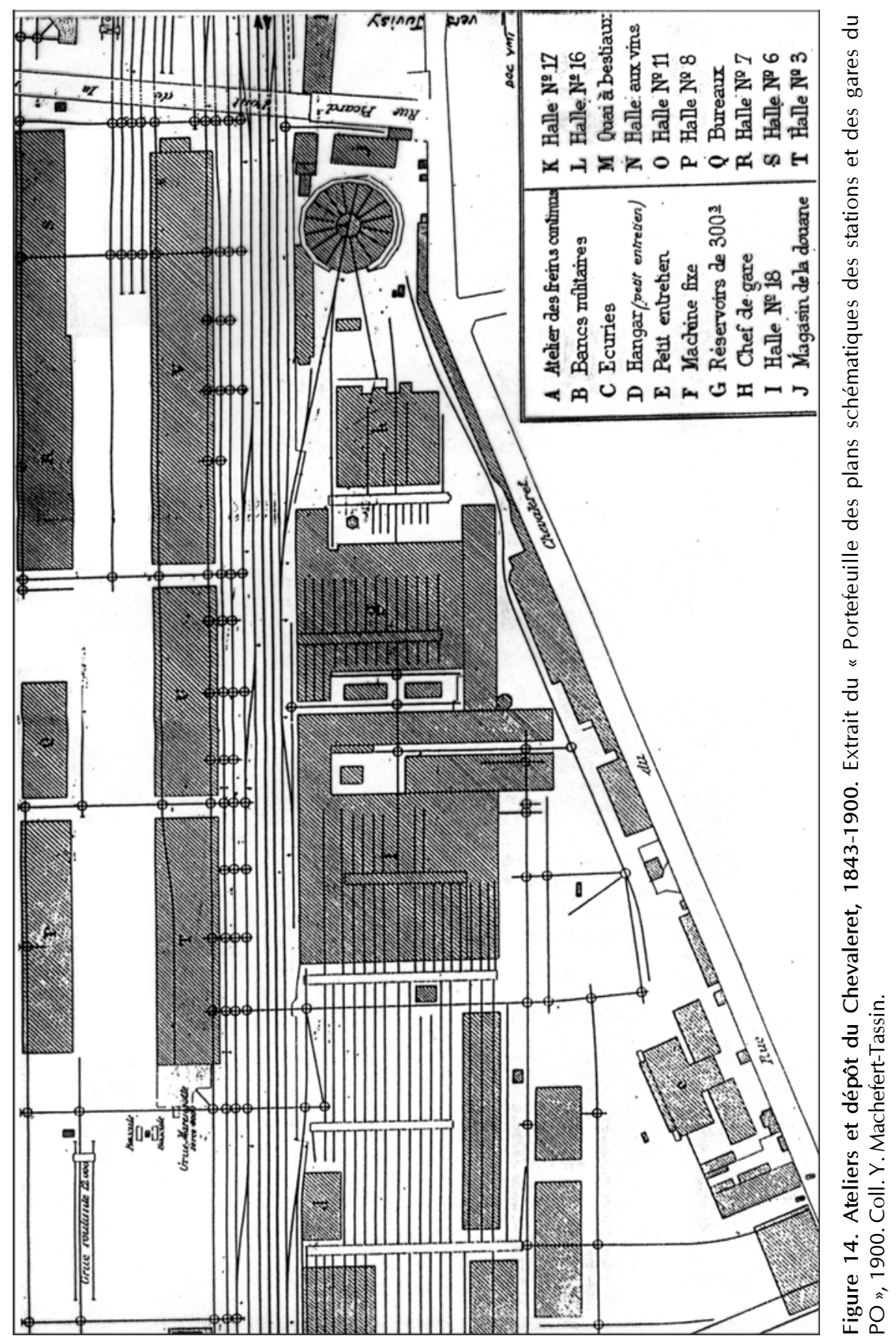




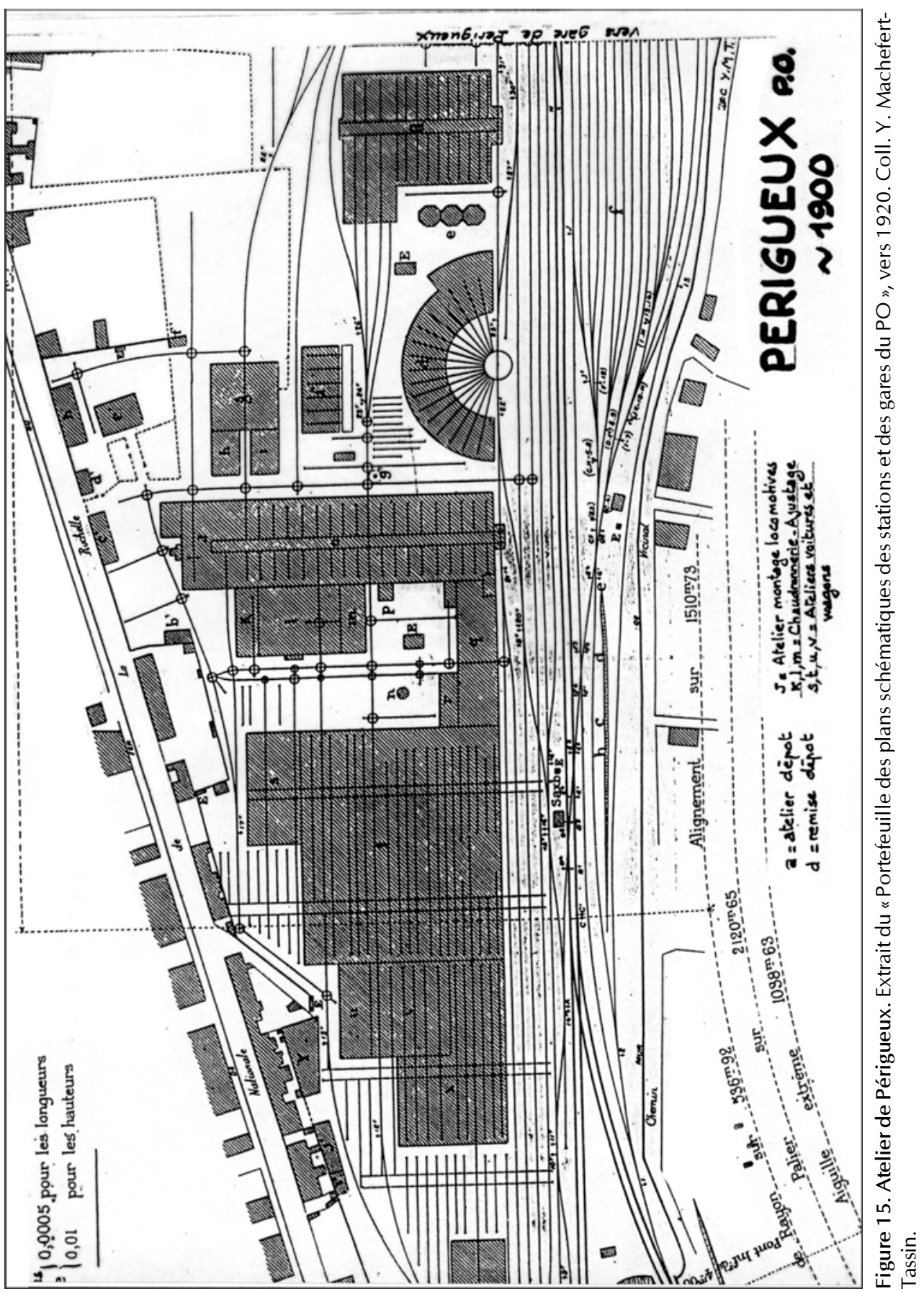




\section{La Compagnie des chemins de fer PLM}

Le PLM avait des ateliers à Nîmes (de 1856 à 1858 - construction de 1863 à 1914), en Arles (de 1898 à 1914), à Oullins par le rachat des ateliers Parent et Schaken (1863-1914), à Paris-Charolais (de 1869 à 1909) (figure 16).

Dans ces ateliers furent construites 1041 unités, soit la presque intégralité du parc de traction de la compagnie jusqu'en 1910 environ. On remarque, en particulier, sous l'impulsion de MM. A. Henry et G. Marié, les $1 \mathrm{~B} 1$ à quatre cylindres Compound C 1 et 2 (prototype) de 1887, construites à Paris ; les 040 à quatre cylindres Compound 4.201 et 202 construites en 1887 à Oullins ; les 120 à quatre cylindres Compound C 3 construites en 1892 à Paris ; les 220 à quatre cylindres, Compound, numérotées C 11/12 en 1892 à Paris et dites "petites $C$ »; enfin, les 220 quatre cylindres Compound, numérotées C 21 à 30, construites en 1894 à Paris puis en Arles en 1900 et dites " grosse C » ou «coupevent » (fig. 21).

Puis on se doit de signaler la construction en 1909, sous l'autorité de M. Chabal, des deux prototypes « Pacific » $n^{\circ}$ 6001, 231 Compound à quatre cylindres à vapeur surchauffée et $n^{\circ}$ 6002, 231 à simple expansion, à 4 cylindres et vapeur surchauffée afin de mener des recherches parallèles sur des locomotives à grandes vitesses. Un grand nombre de locomotives furent reconstruites ou transformées, par exemple la 241 A en 241 D. De même les 1 B1 Compound quatre cylindres de la série 111-400 construite de 1879 à 1884 furent transformées sous plusieurs formes : 220, 130, 040, 040 T, 031 à tender séparé.

Une remarque s'impose : les rames aérodynamiques reçurent des 221 de 1907 modernisées aux ateliers d'Oullins en 1934 qui effectuaient en 1935 le service Paris-Lyon en 5 h 14, avec une vitesse maximum relevée de 120 à $140 \mathrm{~km} / \mathrm{h}$. 


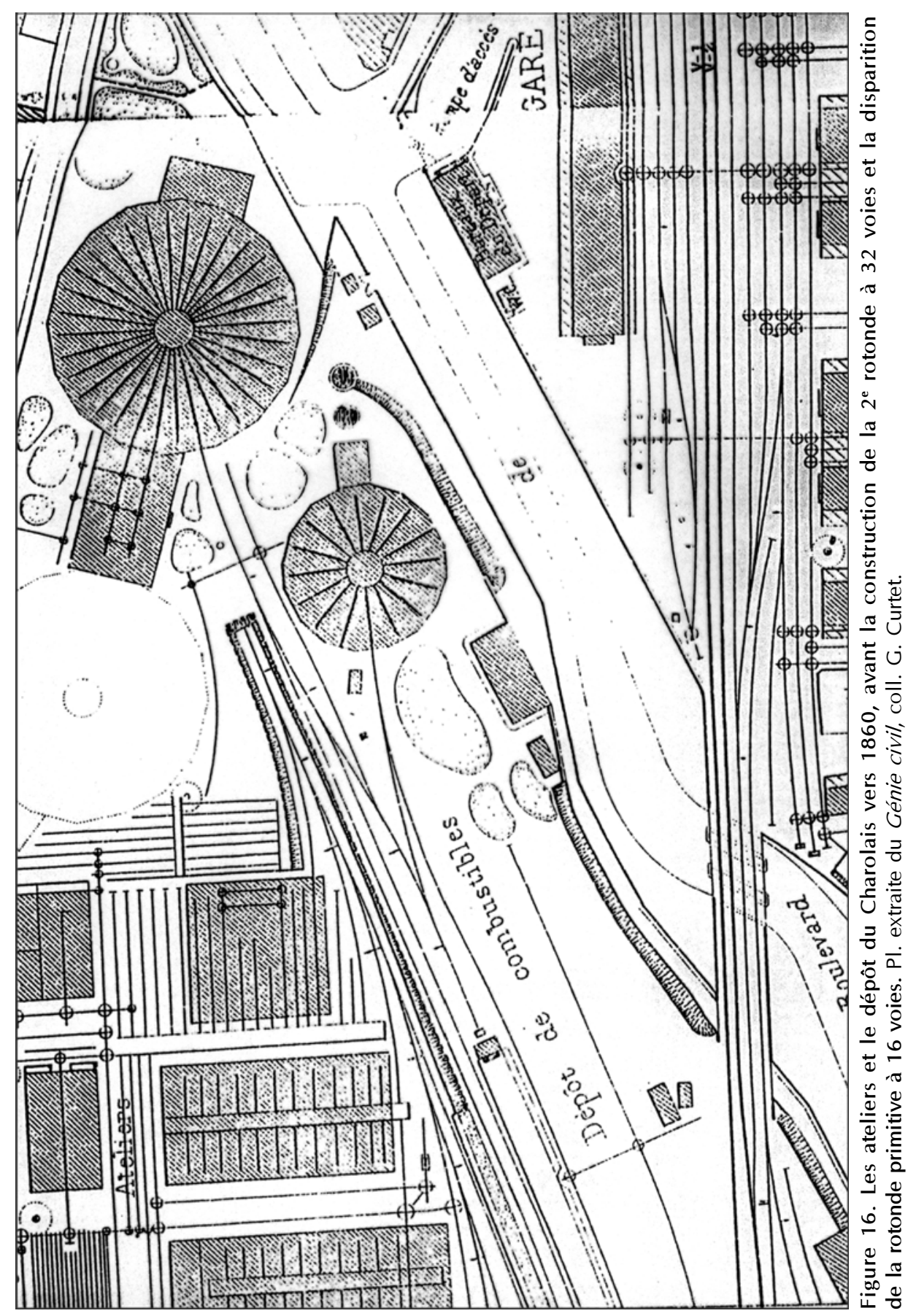




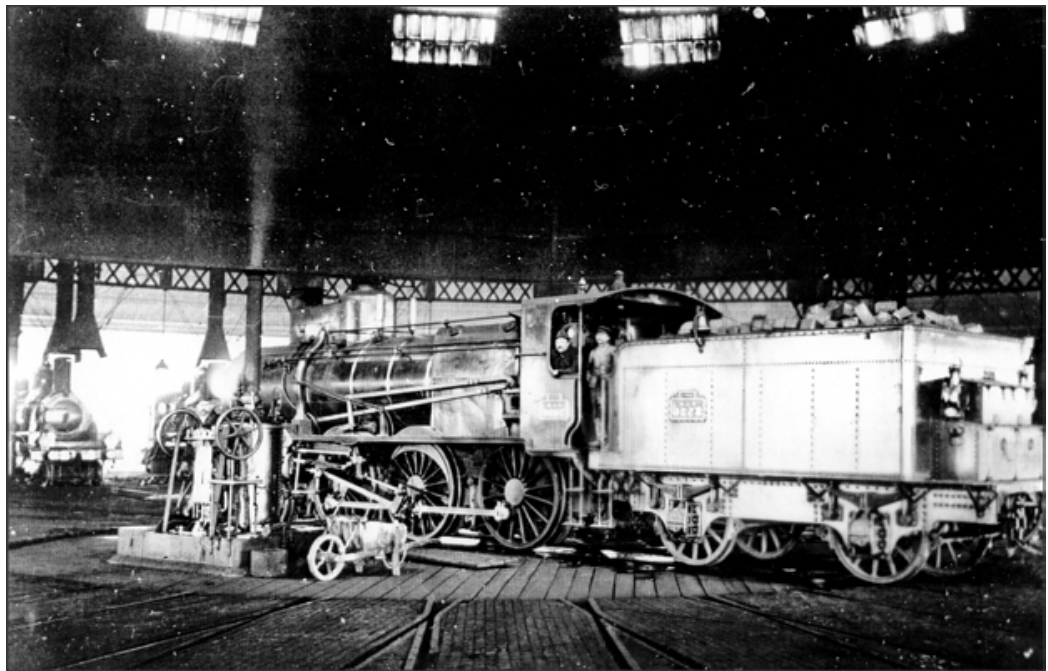

Figure 17. Une rotonde du PLM comme celle de Nevers ou, plus tard, de Chambéry, couverte, avec une $230 \mathrm{~A}$. La photographie a l'avantage de montrer le plancher complet de la fosse de la plaque tournante. La machine à vapeur qui manœuvrait l'appareillage était une particularité du PLM. Cl. PLM, coll. G. Curtet.

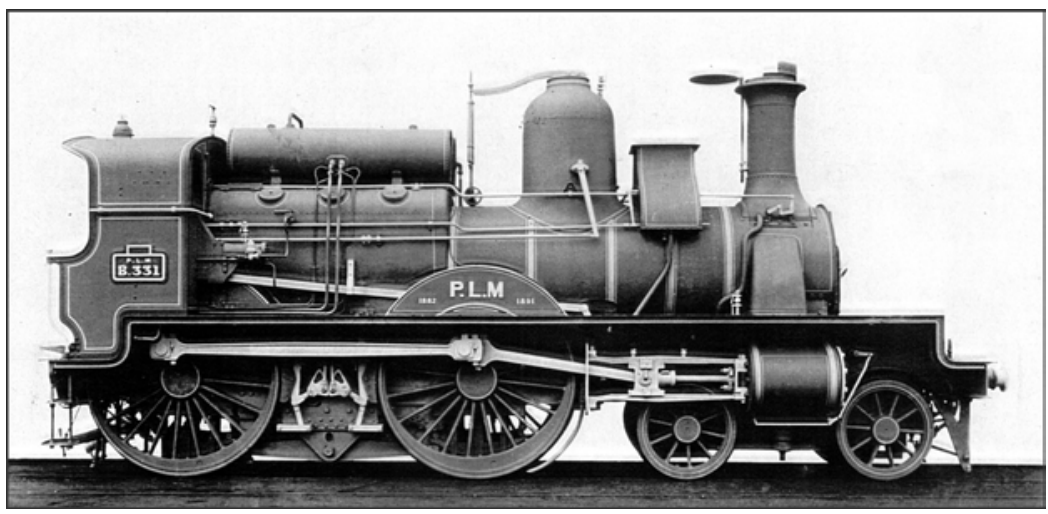

Figure 18. Locomotive PLM 220-331, type B, construite en 1891 à Paris (simple expansion, vapeur saturée, deux cylindres, avec tubes Serve et bogie). Cl. PLM, coll. G. Curtet. 


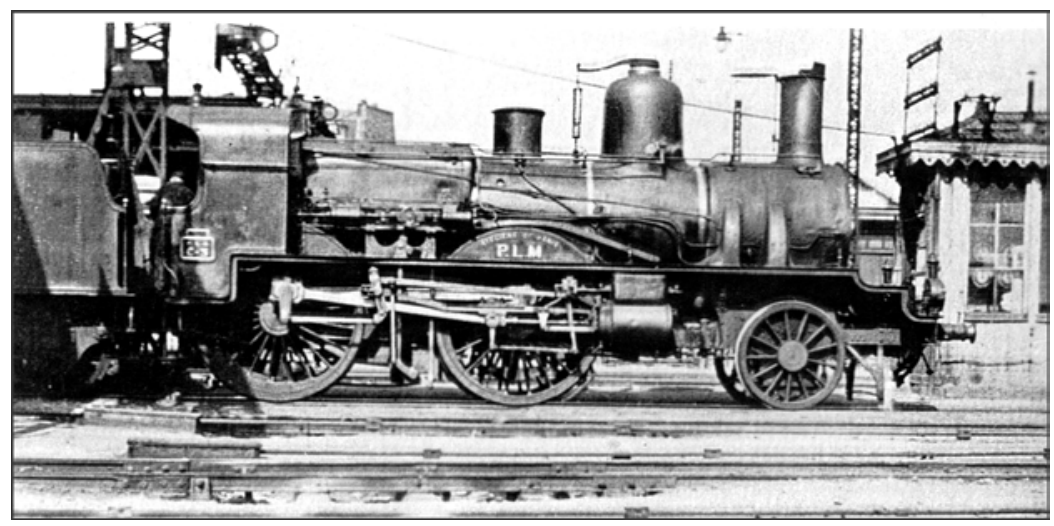

Figure 19. Locomotive 120 type $\mathrm{C} 3$, prototype Compound à quatre cylindres étudié par A. Henry, construite en 1892 à Paris. Cl. Fohano. Coll. L.M.V.

\section{La Compagnie des chemins de fer du Midi}

Ses ateliers situés à Bordeaux Saint-Jean (1857-1918/1920) ne construisent qu'une série de machines neuves. Mais, sous l'influence de M. A. Herdner, le parc de traction fut constamment en évolution. Les ateliers réalisant de nombreuses modernisations, la compagnie fut amenée à prévoir un atelier nord (Bordeaux) et un atelier sud (Béziers) dont une partie sera ensuite revendue en 1920 à la Société Fouga, pour effectuer des révisions générales de locomotives à vapeur.

À Bordeaux-Saint-Jean, le dépôt initial des locomotives, avec une grande remise annulaire, de situait au milieu des ateliers ; mais il fut transféré vers le Sud-Est et séparé d'eux dans les années 1900. De même, à Béziers, où à l'origine dépôt et atelier général étaient réunis, le site devint surtout un dépôt, avec la création de nouveaux ateliers plus au Sud. Plus tard, avec l'électrification Béziers-Neussargues, l'atelier au nord des voies principales fut surtout consacré aux nouvelles machines, tout en restant un dépôt mixte, vapeur et électrique. Dans le même temps les Ateliers de Bordeaux devenaient les ateliers généraux des autorails et l'ancienne rotonde était transformée en magasin. Le dépôt en était alors totalement séparé et devait effectuer sur les locomotives des opérations d'entretien proches des révisions les plus importantes.

\section{Conclusion}

Ce panorama et les exemples choisis montrent bien que, sur la totalité des réseaux français et dès leurs origines, des ateliers généraux regroupaient wagons, voitures et locomotives à vapeur et coexistaient 
avec des dépôts qui étaient souvent parmi les plus importants du réseau. Cette promiscuité offrait des avantages d'un point de vue matériel comme humain : d'une part l'atelier, souvent unique aux origines des compagnies, traitait à la fois le gros entretien et les révisions des engins de traction, d'autre part le dépôt trouvait dans le personnel d'atelier des agents de traction supplémentaires pour l'aider à passer les grandes pointes de trafic. Réciproquement, en période creuse, l'effectif des ouvriers affectés à l'entretien et aux réparations était augmenté.

Cependant cette organisation avait une limite, spatiale avant tout : les surfaces disponibles pour les ateliers intra-muros de Paris (La Chapelle au Nord, La Villette à l'Est, le Charolais au PLM, Le Chevaleret surnommé Ivry au PO, Vaugirard et surtout Batignolles à l'Ouest) devinrent vite insuffisantes avant la fin du XIX ${ }^{e}$ siècle pour développer simultanément les deux activités. Le transfert en banlieue ou en province de la plupart des ateliers intervint dès 1889 (de La Villette vers Noisyle-Sec et vers Épernay, d'« Ivry » vers Tours et vers Périgueux, du Charolais vers Nevers) alors que les dépôts de locomotives restaient logiquement sur place, dotés d'un reste d'atelier d'entretien. Certains furent néanmoins déplacés ou partagés (La Villette avec Noisy, La Chapelle avec La Plaine, Vaugirard à Montrouge, etc.).

Ces déplacements n'étaient toujours du goût des bureaux de conception de locomotives à vapeur et de leurs ingénieurs qui voyaient s'éloigner de leur tutelle et de leur voisinage les moyens de contrôle et d'expérimentation de leurs réalisations, mais ce ne fut pour eux qu'une gêne passagère dans la mesure où les transferts s'étaient accompagnés d'améliorations considérables apportées aux bâtiments d'ateliers et de la rénovation de leur outillage. Nous évoquerons deux exemples opposés de ces évolutions : un contre-exemple d'abord, celui du transfert de l'atelier du PLM à Nevers, dont la construction entreprise tardivement, en 1914, pour remplacer l'atelier parisien n'est pleinement terminée qu'en 1918. On lui affecte le montage et l'entretien des locomotives du Transportation Corps américain, avant que les installations ne soient confiées à l'industrie privée (CGCM) en 1920. Ce n'est que plus tard que le PLM puis la SNCF récupèrent l'atelier. À Béziers en revanche continuent de coexister dépôt et atelier - un atelier qui, de régional, devient général, complément pour les machines électriques de l'atelier de dépôt de Vitry.

Il ne s'agit plus alors de vapeur et, après 1940, les ateliers ne transforment plus comme auparavant et construisent encore moins ces merveilleuses machines à vapeur qui furent leur première raison d'être : un motif, s'il en était besoin, pour rendre hommage aux locomotives et à leurs concepteurs. 


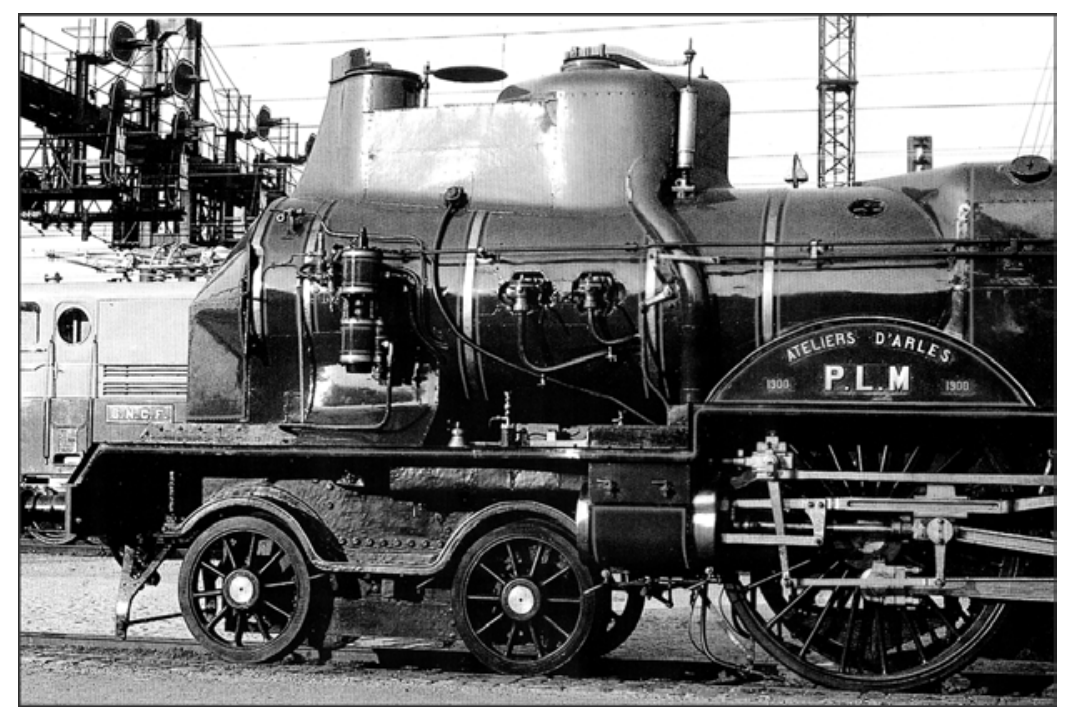

Figure 20. Pour rappeler I'œuvre des Ateliers d'Arles voici I'image, parlante pour tous les cheminots, de la machine PLM - Grosse C - qui se trouve aujourd'hui au Musée français du chemin de fer (220, dite "Coupevent ", 1900). Cl. G. Curtet. 